\title{
How States Become Fragile: Relevancy of State Fragility and Climate Change
}

\author{
Sirui Zhang ${ }^{1, \bullet}$, Jiebin Chen ${ }^{2, \bullet}$,Hao-Ning $\mathrm{Wu}^{2}$ and Shiqi $\mathrm{Ye}^{2, *}$ \\ ${ }^{1}$ Department of Finance, Jinan University, 511400 Guangzhou, China \\ ${ }^{2}$ Department of Mathematics, Jinan University, 511400 Guangzhou, China
}

\begin{abstract}
The objective of this article is to develop a main index that is capable of determining a state's fragility and simultaneously measuring the impact of climate change. Six indexes are defined including a dummy one, which are Political Conditions, Economic Conditions, Social Conditions, Demographic Factor, Public Security and State Unrest (dummy). Five non-dummy indexes are involved four sub-indexes respectively. The sum of these six indexes is reported as State Stability Index (SSI). Entropy method and group decision making serve as weighting models to determine weights of sub-indexes and indexes respectively. A state's fragility is determined by State Fragility Index (SFI), which is the reciprocal of SSI. Five non-dummy indexes and SFI have their standards obtained by K-means, in order to determine whether a state is fragile, vulnerable or stable.
\end{abstract}

\section{Introduction}

There exists some methodology or models to determine a state's fragility, for instance, the well-known Fragile State Index [1], fuzzy method [2], empirical strategy of Graziella Bertocchi and Andrea Guerzoni [3] and so on. In this paper, we shall define State Fragility Index (SFI), determining fragility of states by AHP (Analytic Hierarchy Process) based on entropy method and group decision making.

We define 20 sub-indexes to construct 5 indexes (1 index is related to 4 sub-indexes), Political Conditions (PC), Economic Conditions (EC), Social Conditions (SC), Demographic Factor (DF) and Public Security (PS). A dummy index State Unrest (SU) is also considered. Then State Stable Index (SSI) is defined based on the six indexes. SFI takes its value as $1 / \mathrm{SST}$. The weights of 20 sub-indexes are determined by the entropy method objectively. The weights of five indexes are determined by group decision making, which consists of four experts. By normalizing the vector

$\sum_{i=1}^{4} \lambda_{i} W^{(i)}=\lambda_{1} W^{(1)}+\lambda_{2} W^{(2)}+\lambda_{3} W^{(3)}+\lambda_{4} W^{(4)}$

Where $\lambda_{i}, W^{(i)}$ are the largest eigenvalue and its corresponding eigenvector of the $i^{\text {th }}$ pairwise comparison matrix, we obtain the weights vector $\mathrm{W}^{*}$ for the six indexes. We shall then consider the standard of evaluating whether a state is fragile, vulnerable or stable. All indexes have their standards, and so does SFI. These standards are obtained by k-means. One can compare a certain state with these standards.

Based on Climate Evaluation Index (CEI) we shall define, one can measure climate change's impact on state fragility. Furthermore, we shall present an explicit expression for the impact based on our model. When value of CEI increase $1 \%$, SFI shall increase $0.311 \% \times h$, where $h$ is unique for a certain state, obtained by normalizing all states' Environmental Performance Index (EPI).

We consider Malaysia as a case, evaluating its state fragility and determine the way and extent that climate change may impact on them based our model. Then a specific time series analysis of Malaysia shown that in 2000, Malaysia converts fragility into vulnerability. And it stays in the band of vulnerability since 2000 .

Finally we shall consider the modification of our model, enhancing its applicability. Evaluation of our model is also given, inclusion strength, weakness and sensitivity analysis.

The rest of the paper is organized as follows. In Section 2, we shall define the model and give some theoretical results. Case study of Malaysia is given in Section 3. The modification of the model and future work can found in Section 4.

\section{Methodology}

In this section, we shall consider the whole methodology. The index system is constructed firstly, consisting of 5 indexes and 18 sub-indexes. The weights of sub-indexes are determined by the entropy method (for some other applications, see $[4,5,6])$, while the weights of indexes are determined by group decision making. Hence we built the evaluation for state fragility. The measurement of climate change impact on state fragility and identification of its means and fragility of a state shall be completed by K-means.

\footnotetext{
- These authors contribute equally to this work. " Corresponding author: ojwlg@jnu.edu.cn.
} 


\subsection{Index System}

We define State Fragility Index (SFI) to measure state fragility. To construct SFI, we first consider State Stability Index (SSI) and then SFI $=1 /$ SSI. More details are shown in a moment.

Table 1. Table of sub-indexes

\begin{tabular}{|l|l|l|l|}
\hline Sub-index & Abbr. & Index & Source \\
\hline State legitimacy index & SLI & & FFP \\
Corruption perception index & CPI & \multirow{2}{*}{ PC } & TI \\
Human Rights\&Rule of Law & HRL & & FFP \\
Democracy index & DEI & & Economist \\
\hline GDP(PPP)per capita & GDP & & DataBank \\
Index of Economic Freedom & IEF & EC & FI \\
Gini coefficient & GNC & & Data Bank \\
Inflation rate & IFR & & CIA \\
\hline World happiness index & WHI & & UNSDSN \\
Lift expectancy index & LEI & & DataBank \\
Education index & EDI & & UNDP \\
Global gender gap & GGG & & WEF \\
\hline Population density & PPD & & DataBank \\
Net immigration rate & NMR & DF & CIA \\
Infant mortality rate & IMR & & CIA \\
Global hunger index & GHI & & IFPRI et.al. \\
\hline Intentional homicide rate & IHR & & UNODC \\
Number of guns per capita & ENG & PS & GGIIS \\
Global peace index & GPI & & IEP \\
Global terrorism index & GTE & & IEP \\
\hline
\end{tabular}

Six indexes are defined to evaluate SSI, which are Political Conditions (PC), Economic Conditions (EC), Social Conditions (SC), Demographic Factor (Population conditions, denoted as DF), Public Security (PS) and a dummy index State Unrest (SU). SU shall involve some unpredictable factors, such as war, nationwide strike and so on. SU takes its value in $\{0,1\}$, it takes value 0 when the state is in unrest or it shall take 0 (remember we are considering SST!). The evaluation result, the fragility of a state, is expressed as State Fragility Index (SFI). There also exists 20 sub-indexes determining the 5 indexes, which are shown in Table 1.

\subsection{Weight Determination Based on Entropy Method and Group Decision Making}

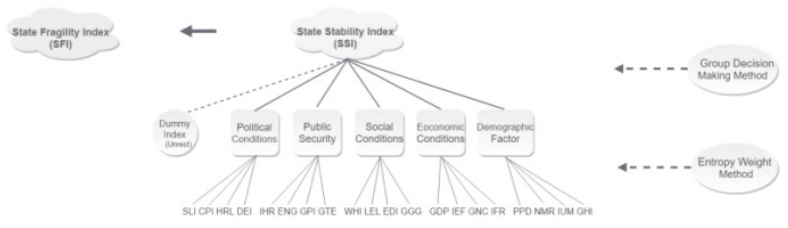

Fig. 1. Weight Determination Model Diagram. The Diagram illustrates that weight determination model consists of two method, then entropy method and group decision making.

\subsubsection{Weight Determination Based on Entropy Method}

We first consider the weights of 20 sub-indexes belonging to 5 indexes. Let $X$ be the e fuzzy evaluation matrix (see [7]) with element

$$
X_{i j}, \quad i=1,2, \ldots, m, j=1,2, \ldots, n,
$$

where $i, j$ denotes the $i^{\text {th }}$ indicator and the $j^{\text {th }}$ state respectively. Then the steps of entropy method are as follows [6]:
1. Normalization. For those indicators which are better when their values are larger, they are normalized by

$$
X_{i j}^{*}=\frac{X_{i j}-\min \left\{X_{i 1}, X_{i 2}, \ldots, X_{i n}\right\}}{\max \left\{X_{i 1}, X_{i 2}, \ldots, X_{i n}\right\}-\min \left\{X_{i 1}, X_{i 2}, \ldots, X_{i n}\right\}} .
$$

And for the others (the smaller values are, the better they are), the formula is

$$
\mathrm{X}_{\mathrm{ij}}^{*}=\frac{\max \left\{\mathrm{X}_{\mathrm{i} 1}, \mathrm{X}_{\mathrm{i} 2}, \ldots, \mathrm{X}_{\mathrm{in}}\right\}-\mathrm{X}_{\mathrm{ij}}}{\max \left\{\mathrm{X}_{\mathrm{i} 1}, \mathrm{X}_{\mathrm{i} 2}, \ldots, \mathrm{X}_{\mathrm{in}}\right\}-\min \left\{\mathrm{X}_{\mathrm{i} 1}, \mathrm{X}_{\mathrm{i} 2}, \ldots, \mathrm{X}_{\mathrm{in}}\right\}} .
$$

We still denote the normalized indicator $X_{i j}^{*}$ as $X_{i j}$.

2. Calculation of the ratio of each indicator by

$P_{i j}=\frac{X_{i j}}{\sum_{j=1}^{n} X_{i j}}, \quad i=1,2, \ldots, m, j=1,2, \ldots, n$.

3. Calculation of the entropy value shall be obtained by

$$
e_{i}=-k \sum_{j=1}^{n} P_{i j} \ln P_{i j}
$$

where $i=1,2, \ldots, m, j=1,2, \ldots, n, \mathrm{k}=\frac{1}{\ln n>0}$ and suppose when $\mathrm{P}_{i j}=0$, then $\mathrm{P}_{i j} \ln \mathrm{P}_{i j}=0$.

4. Definition of the entropy weight. The entropy weight of the $i^{\text {th }}$ indicator shall be defined as

$$
\begin{gathered}
w_{i}=\frac{1-e_{i}}{m-\sum_{i=1}^{m} e_{i}}, \quad i=1,2, \ldots, m \\
\text { in which } 0 \leq \omega_{\mathrm{i}} \leq 1, \sum_{i=1}^{m} \omega_{\mathrm{i}}=1
\end{gathered}
$$

\subsubsection{Weighting Model Based on Group Decision Making}

Hence we have determined the weights of sub-indexes to their corresponding index, we shall consider the weights of five indexes by group decision making, which is a particular application of AHP [8]. The expert group consists of four experts who give a pairwise comparison matrix independently like

$$
A=\left(A_{i j}\right), i=1,2, \ldots, 6, j=1,2, \ldots, 6 .
$$

Each matrix shall pass consistency check, which is stated as follows.

$$
C I=\frac{\lambda-n}{n-1}, \quad C R=\frac{C I}{R I},
$$

where $\lambda$ is the largest eigenvector of the pairwise comparison matrix and $n=4$ here. The matrix passes consistency check if $C R<0: 1$. Values of RI can be found in Table 2 [8].

Table 2. Random Consistency Index (RI)

\begin{tabular}{|c|ccccccccccc|}
\hline $\mathrm{n}$ & 1 & 2 & 3 & 4 & 5 & 6 & 7 & 8 & 9 & 10 & 11 \\
\hline $\mathrm{RI}$ & 0 & 0 & 0.58 & 0.90 & 1.12 & 1.24 & 1.32 & 1.41 & 1.45 & 1.49 & 1.51 \\
\hline
\end{tabular}

The normalized corresponding eigenvector of $\lambda$ gives the weights of indexes. Here we have four eigenvalues and four normalized corresponding eigenvector.

$$
W^{(i)}, \quad i=1,2,3,4 \text {. }
$$

Then the weights of six indexes shall be obtained by normalizing the following vector:

$\sum_{i=1}^{4} \lambda_{i} W^{(i)}=\lambda_{1} W^{(1)}+\lambda_{2} W^{(2)}+\lambda_{3} W^{(3)}+\lambda_{4} W^{(4)}$. and we denote the result as $\mathrm{W}^{*}$.

\subsection{State Fragility Index}

State Fragility Index (SFI) shall be constructed as follows:

1.Determination of weights of the six indexes. For each expert, we obtain a pairwise comparison matrix, 
which is scored by the importance among these indexes and experts' experiences as follows:

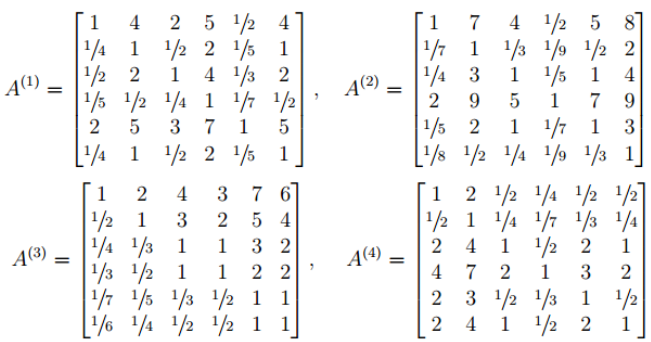

Their CR values are $0.0087,0.0246,0.0252$ and 0.0082 , i.e., they all pass the consistency check. Then the weights for six indexes shall be obtained by normalizing vector:

$W^{*}=\{0.2006,0.1603,0.1706,0.1101,0.2043,0.1541\}$.

2. Construction of State Stability Index (SSI). SSI shall be calculated by

$$
\begin{aligned}
& S S I=W_{1}^{*} P C+W_{2}^{*} E C+W_{3}^{*} S C+W_{4}^{*} D F+ \\
& W_{5}^{*} P S+W_{6}^{*} S U=0.2006 P C+0.1603 E C+ \\
& 0.1706 S C+0.110 D F+0.2043 P S+0.1541 \mathrm{~S}
\end{aligned}
$$

where $S U$, a dummy index taking value in $[0,1]$, represents state unrest.

3. Construction of State Fragility Index (SFI). Every sub-indexes are normalized into $[0,1]$, their corresponding indexes also take values in $[0,1]$ because of $\sum_{i=1}^{m} \omega_{i}=1$. So does the expression

$W_{1}^{*} P C+W_{2}^{*} E C+W_{3}^{*} S C+W_{4}^{*} D F+W_{5}^{*} P S+W_{6}^{*} S U$.

Then the formula SFI $=\frac{1}{S S T}$ can evaluate the fragility of a state.

\subsection{Identification of State Fragility by K-means}

We use K-means to partition some states into 3 part as to index respectively, i.e., we use it five times. Thus we obtain five pairs of critical values, which are shown in Figure 3. Because of data miss, we cluster different number of states as to different index.
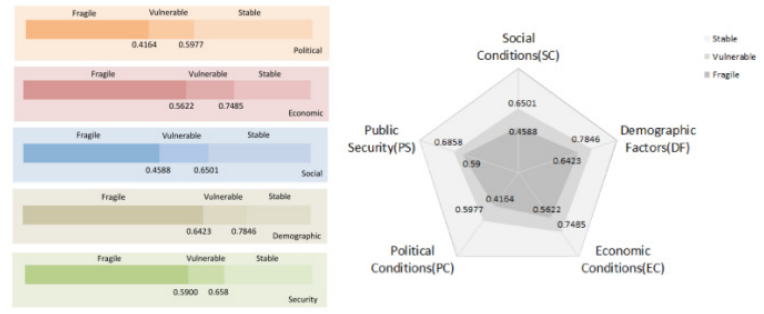

Fig. 2. Standard of Identifying State Fragility with Respect to the Five Indexes. These plots illustrate the standard of identifying fragility of a state. The left plot shows the critical values of different indexes. And the right radar diagram shows the fragility of a certain state with respect to the five indexes.

\subsection{Measurement of Climate Change's Impact and Identification of Its Means}

We shall construct Climate Evaluation Index to evaluate climate conditions. Then we consider how climate change impacts fragility of a state.

\subsubsection{Climate Evaluation Index}

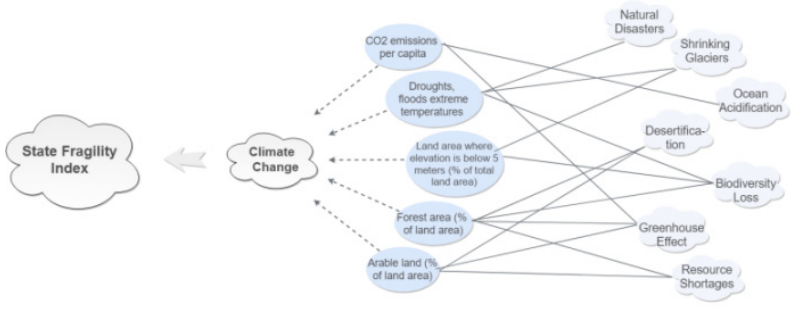

Fig. 3. Climate Evaluation Index Diagram. The diagram illustrates the construction of CEI and its connection to some results of climate change.

Climate Evaluation Index (CEI) consists of CEPC $\left(\mathrm{CO}_{2}\right.$ emissions per capita), DFET (Droughts, floods, extreme temperatures (\% of population)), LABF (Land area where elevation is below 5 meters ( $\%$ of total land area)), FRLD (Forest area ( $\%$ of land area)) and ARAR (Arable land (\% of land area)), involving natural disasters, shrinking glaciers, ocean acidification, desertification, bio-diversity loss, greenhouse effect, resource shortage, etc (See Figure 4). The weights of these five indexes are determined by the entropy method mentioned above.

\subsubsection{Climate Change's Impact on State Fragility}

Regression analysis helps one understand the relations between a single index in State Fragility Index and a single index in Climate Evaluation Index. We shall focus on the relations among them using regression analysis 25 times ( 5 indexes in SFI $\times 5$ indexes in CEI). Some of them are significant linear ( $p$-value $<0.1$ ), which we shall discuss now, and the others are not significant linear. By regression analysis, we find that there exist 14 pairs of indexes with significant linear relations, they are

$$
\begin{cases}P C=0.26396 L A B F+0.28474, & p_{\text {_value }}=0.021938 \\ E C=-0.28116 L A B F+0.88399, & p_{\text {_value }}=0.010567 \\ S C=-0.34221 L A B F+0.89837, & p_{\text {_value }}=0.0073692 \\ P C=0.3258 C E P C+0.24765, & p_{\text {_value }}=9.2096 \times 10^{-5} \\ E C=-0.5325 C E P C+1.0883, & p_{\text {value }}=1.0177 \times 10^{12} \\ S C=-0.61912 C E P C+1.1243, & p_{\text {_value }}=2.6779 \times 10^{-10} \\ D F=-0.32492 C E P C+1.0262, & p_{\text {_value }}=5.9513 \times 10^{-5} \\ P C=0.20002 D F E T+0.51119, & p_{\text {_value }}=0.00026062 \\ E C=-0.18953 D F E T+0.63702, & p_{\text {_value }}=0.0014249 \\ S C=-0.38207 D F E T+0.6144, & p_{\text {_value }}=1.2051 \times 10^{-7} \\ P C=-0.12652 F R L D+0.57411, & p_{\text {_value }}=0.024171 \\ P S=-0.079467 F R L D+0.65849, & p_{\text {_value }}=0.073205 \\ D F=0.15213 A R A R+0.61821, & p_{\text {_value }}=0.0039786 \\ P S=-0.080755 A R A R+0.68697, & p_{\text {_value }}=0.056034\end{cases}
$$

\subsubsection{Explicit Expression of Climate Change's Impact on State Fragility}

We derive the explicit expression of the climate change's impact on state fragility. When CEI increases $1 \%$, its involving five indexes would also increase $1 \%$. By the system of equations (9), PC shall increase about $0.66 \%$, EC shall decrease about $1 \%$, SC shall decrease about $1.34 \%$, DF shall decrease about $0.17 \%$ and PS shall decrease $0.16 \%$. Hence SSI shall decrease about $0.31 \%$.

Then SFI shall increase $x$, where $x$ shall be solved by 


$$
\operatorname{SFI}(1+x)=\frac{1}{\operatorname{SSI}(1-0.31 \%)} .
$$

Then $\mathrm{x}=\frac{1}{1-0.31 \%}-1 \approx 0.311 \%$. Since effects of climate change vary from state to state, we shall introduce normalized (by Formula 3) Environmental Performance Index (EPI) as a coefficient to measure the variation, denoted as $h$. Hence we obtain the explicit expression of climate change's impact on state fragility by this model:

State fragility increases $0.311 \% * h$ if CEI increases $1 \%$.

\section{Case Study: Malaysia}

In this section, we shall consider Malaysia (a coastal state) as a case. Based on the State Fragility Index (SFI), Malaysia' values can be calculated. The SFI value for Malaysia is 1.2811 .

\subsection{Determining the Way and Extent that Climate Change Increases States Fragility}

Determination of the way that climate change may increase states fragility has already been completed in Section 2.5.2. Five indexes of CEI shall impact on the five indexes of SFI respectively.

Since we have obtained the relation between climate change and SFI, we shall determine the extent that climate change may increase states fragility. After normalizing Environmental Performance Index (EPI) by Formula (3), we obtain $h$ value of Malaysia which is 0.2879 . By the explicit expression (9), we know that when CEI increasing $1 \%$, SFI value of Malaysia shall increase $0: 311 \%$ $\times 0.2879 \approx 0.0895 \%$.

Remark 1. Note that the increase extent is for the State Fragility Index (SFI), not the real state fragility.

Remark 2. Definitive indicators which push states to become more fragile have been considered in Section 2.5.2.

\subsection{Time Series Analysis on Malaysia and the Tipping Point}

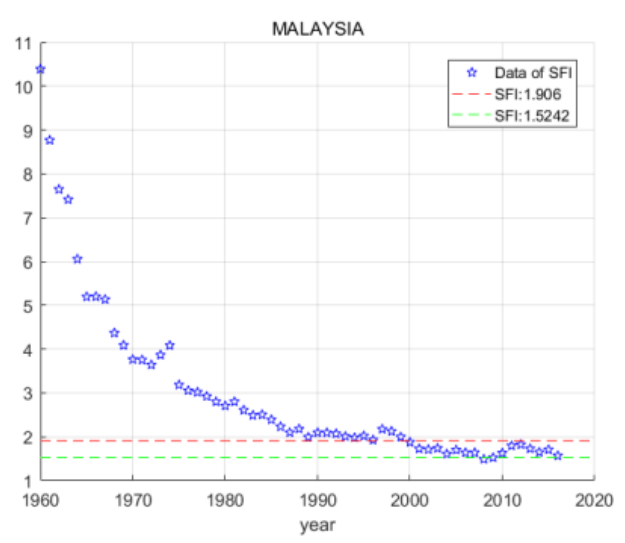

Fig. 4. Time Series Analysis of SFI on Malaysia. The plot illustrates that SFI value of Malaysia is decreasing in this year.
We now consider time series of SFI on Malaysia ranging from 1960 to 2016, see Figure 4. The tipping points, i.e. the standards mentioned above, are also shown in the plot. We find that in 2000, Malaysia converts fragility into vulnerability. And it stays in the band of vulnerability since 2000.

\section{Modification and Future Work: A More General Model}

Will our model work on smaller states (such as cities) or larger states (such as continents)? It's hard to answer this question only by our model. We shall compare our model with works of others, such as the well-known Fragile State Index (FSI) produced by the Fund of Peace [1]. We shall pick up two states from our model, Haiti and India. Haiti shall be deemed as a smaller case, whose total area is $27,750 \mathrm{~km}^{2}$, almost equal to the sum of Beijing and Tianjin (two Chinese cities). And India, the 7th largest state in the world, shall be deemed as a larger case. In our SFI, India is ranked 5th out of 75 states, while it is ranked 72 nd out of 178 states in FSI. And Haiti is ranked 33rd out of 75 state in our SFI, but it is ranked 11 th out of 178 states. Both states illustrate that our model may not work on smaller or larger states, let along cities or continents.

Hence we shall modify our model. Collecting more data may be a method. Due to the data scarcity, we ranked only 75 states by our model. Results may be better when we obtain full data of different index and different states. Another method is to introduce a coefficient into our model based on the "scale" of state. The value of SFI shall multiply by a certain coefficient to adjust the results. The definition of the coefficient shall be a future work.

\section{References}

1. The Fund for Peace. Fragile states index 2017, 2017. http://fundforpeace.org/fsi/data/.

2. Mina Baliamoune-Lutz and Mark McGillivray. Fragile states: causes, costs, and responses, Chapter State fragility: Concept and measurement, pages 3342. Oxford University Press New York, 2011.

3. Graziella Bertocchi and Andrea Guerzoni. Growth, history, or institutions: What explains state fragility in sub-saharan africa? Journal of Peace Research, 49(6):769-783, 2012.

4. Raid Al-Aomar. A combined ahp-entropy method for deriving subjective and objective criteria weights. International Journal of Industrial Engineering: theory, applications and practice, 17(1), 2010.

5. Hongliang Zhao, Leihua Yao, Gang Mei, Tianyu Liu, and Yuansong Ning. A fuzzy comprehensive evaluation method based on ahp and entropy for a landslide susceptibility map. Entropy, 19(8):396, 2017.

6. Zhi-Hong Zou, Yun Yi, and Jing-Nan Sun. Entropy method for determination of weight of evaluating indicators in fuzzy synthetic evaluation for water quality assessment. Journal of Environmental 
Sciences, 18(5):1020-1023, 2006.

7. Lotfi A Zadeh. Fuzzy sets as a basis for a theory of possibility. Fuzzy sets and systems, 100:9-34, 1999.

8. Thomas L Saaty and Kirti Peniwati. Group decision making: drawing out and reconciling differences. RWS publications, 2013. 